\title{
Simple Binary Prediction of Daily Storm-Level Geomagnetic Activity with Solar Winds and Potential Relevance for Cerebral Function
}

\author{
Joseph M. Caswell, Nicolas Rouleau \\ Transnational Anomalies Research, Sudbury, Ontario P3E 3W6, Canada \\ E-mail addresses: neuraljc@gmail.com ,ny_rouleau@laurentian.ca
}

\begin{abstract}
A number of previous studies have examined various statistical methods for the prediction of geomagnetic activity, particularly based on predictor input of solar wind variables. However, investigation of the potential for a simple binary prediction system based on either "quiet" or stormlevel activity of the planetary magnetic field has been severely lacking. The goals of the current analyses were to identify potential space weather models for the accurate prediction of geomagnetic storm events. Furthermore, while the deleterious or negative effects of increases in geomagnetic activity on a range of terrestrial systems have been focused on in the past, theoretical perspectives on the potential benefits of significantly increased geomagnetic perturbations are considered.
\end{abstract}

Keywords: Geomagnetic field; solar winds; interplanetary magnetic field; prediction; logistic regression; artificial neural network; space weather

\section{INTRODUCTION}

The geomagnetic field of our planet has been previously associated with a wide range of terrestrial effects on electrical systems [1], weather conditions [2], and human health [3]. It has also been shown that even small geomagnetic changes in the range of $20-40 \mathrm{nT}$ are associated with neuroelectrical perturbations which may produce altered states of consciousness [4], suggesting neural correlates of consciousness must therefore be examined within the context of extra-planetary forces. The prediction of geomagnetic disturbances has a range of implications for activity on Earth, with many previous statistical applications employed for this purpose [5-6]. However, there are very few studies which have examined the potential for a simply binary prediction of storm-level geomagnetic events [7], with relatively little statistical detail provided. The primary objective of the current study was to determine the feasibility for simple predictions of binary-coded $(0,1)$ values representing days of either "quiet" or storm-level geomagnetic activity. Furthermore, a wider range of statistical techniques was employed as previously suggested [5].

One of the primary sources of variation of the planetary magnetic field is the Sun itself. While a number of solar-geomagnetic relationships have been identified by earlier researchers [8], the precise nature of these interactions regarding specific components of the solar magnetic field and solar winds remains relatively complicated [9]. As an example, 
previous studies have examined the relationship between variations in the geomagnetic field with solar wind speeds and the interplanetary magnetic field (IMF) [10], which itself is a direct product of the solar magnetic field which has been distributed throughout the heliosphere by solar winds (SW).

Furthermore, other predictive studies of geomagnetic activity have typically employed similar measures of solar activity and SW for model predictors as those used in the current analyses, including variables associated with the interplanetary magnetic field (IMF) [6], solar winds (SW) [5], and overall solar activity [11].

\section{METHODS}

Daily averages of geomagnetic activity ( $A_{p}$ indices) were obtained from the NASA/SPDF OMNIWeb (omniweb.gsfc.nasa.gov/form/dx1.html) for the years 2000 to 2013. This value represents the daily overall level of geomagnetic activity averaged across a number of observatories, with "storm-level" activity occurring when $A_{p}>29$. A nominal binary variable was computed for $A_{p}$ indices based on the presence or absence of daily geomagnetic storm activity. There were 277 geomagnetic storm cases for which additional predictor data were also available in full. A sample of 300 geomagnetically "quiet" days $\left(\mathrm{A}_{\mathrm{p}}\right.$ $<30$ ) was randomly selected from the total database for comparison (total of 577 cases).

Additional variables were also obtained from the NASA OMNIWeb for daily averages of the scalar component of the interplanetary magnetic field (IMF), proton density of solar winds (SW), SW plasma speed, SW plasma flow pressure and solar radio flux. Data for cosmic ray (CR) impulses were acquired from the online Moscow Neutron Monitor database (cr0.izmiran.rssi.ru/mosc/main.htm). Measures and descriptive statistics for all space weather variables are displayed in Table 1.

Table 1. Units of measurement and descriptive statistics for 577 daily average cases of each predictor variable examined; $\mu=$ mean, $\sigma=$ standard deviation, $\mathrm{SEM}=$ standard error of the man, $\min =$ minimum value, $\max =$ maximum value.

\begin{tabular}{|c|c|c|c|c|c|c|}
\hline \multicolumn{1}{|c}{ Variable } & Measurement Units & $\boldsymbol{\mu}$ & $\boldsymbol{\sigma}$ & \multicolumn{1}{c|}{ SEM } & \multicolumn{1}{c|}{ min } & max \\
\hline Scalar IMF & $\mathrm{nT}$ & 8.011 & 4.328 & .18 & 2.1 & 33.4 \\
\hline Proton Density & $\mathrm{N} \cdot \mathrm{cm}^{-3}$ & 6.273 & 4.126 & .172 & .5 & 30 \\
\hline Plasma Speed & $\mathrm{km} \cdot \mathrm{s}^{-1}$ & 497.815 & 129.248 & 5.381 & 256 & 1003 \\
\hline Flow Pressure & $\mathrm{nPa}$ & 3.005 & 2.362 & .098 & .3 & 17.44 \\
\hline Radio Flux & $10^{-22} \mathrm{~W} \cdot \mathrm{m}^{-2} \cdot \mathrm{Hz}^{-1}$ & 125.529 & 46.276 & 1.927 & 66.2 & 325.1 \\
\hline Cosmic Rays & Mean \# of impulses per minute & 8683.28 & 557.345 & 23.243 & 6714 & 9862 \\
\hline
\end{tabular}

Logistic regression, receiver operating characteristics, and mean comparison analyses were conducted using SPSS 17, while Matlab 2011a software was employed for artificial 
neural network (ANN) testing. Sensitivity analysis of regression coefficients (odds-ratio; OR) and all measures of statistical power were verified using the $G^{*}$ Power 3 application [12-13].

Pseudo- $R^{2}$ values are presented for logistic regression analyses using Nagelkerke's [14] method. This involves acquiring the Cox-Snell pseudo- $R^{2}$ with the relationship 1- $\left(L_{0} / L_{M}\right)^{2 / n}$, where $L_{0}=$ likelihood function for the model prior to predictor entry, and $L_{M}=$ likelihood function for the current model. The upper bound for this value is obtained using 1-[ $P^{P}(1-P)^{(1-}$ $\left.{ }^{P}\right]^{2}$, where $P=$ proportion of event (e.g., geomagnetic storm) cases. Finally, the Cox-Snell value is divided by the upper bound, resulting in the Nagelkerke pseudo- $R^{2}$.

\section{RESULTS}

A binary logistic regression was employed to predict the occurrence of geomagnetic storm-level activity $\left(A_{p}>29\right)$ using backward-conditional variable entry of predictors. SW proton density, solar radio flux, and CR impulses were removed from subsequent models, while the scalar component of the IMF with plasma speed and flow pressure of SW significantly entered the model. Significant predictors were examined for multicollinearity with no issues identified (all variance inflation factors $<10$ ).

Further logistic regression analyses were conducted to facilitate nested comparison of models with the addition of each subsequent predictor variable. The scalar IMF measure entered first (OR $=2.467$, CI $95 \%=2.128-2.861, p<.001)$, revealing a significant overall model $\left(\chi_{(1)}^{2}=422.213, p<.001\right.$, pseudo- $\left.R^{2}=.692\right)$. The plasma speed of SW was then added to the following model which produced a statistically significant change $\left(\Delta \chi^{2}=\right.$ 143.793, $p<.001)$. The addition of SW plasma flow pressure demonstrated a weak odds-ratio $(\mathrm{OR}<2)$, and introduced decreases in OR values for other predictors. As a result, this variable was removed from the final analysis.

The overall model obtained $\left(\chi_{(2)}^{2}=566.006, p<.001\right.$, pseudo- $\left.R^{2}=.834\right)$ consisted of the scalar IMF $(\mathrm{OR}=2.963$, CI $95 \%=2.413-3.639, p<.001)$ and $\mathrm{SW}$ plasma speed $(\mathrm{OR}=$ 1.017 , CI $95 \%=1.013-1.021, p<.001)$. Sensitivity analysis of regression coefficients determined a critical OR value of 1.343 was required. Post-hoc statistical power for the scalar IMF predictor was above the ideal range $(1-\beta>.8)$. However, while statistically significant, the OR obtained for the plasma speed measure presented highly unreliable statistical power $(1-\beta=.053)$. The final model presented with an overall accuracy of $92.2 \%$. No significant effects were observed for the scalar IMF by plasma speed interaction term $(p>.05)$.

To further test these results, a series of artificial neural networks (ANN) were trained to predict the daily geomagnetic storm or no-storm activity. Binary pattern recognition networks were constructed using a single hidden layer with the minimal two hidden neurons, sigmoid (logistic) functions, and the standard Levenberg-Marquardt training algorithm. Initial synaptic weights were randomized.

The scalar IMF and SW plasma speed were entered as input layer predictor neurons. Random samples of $70 \%$ of cases were used to train 1000 individual networks which were validated and tested on the remaining $30 \%$ of cases in each instance. Results from all 1000 networks were averaged to produce greater overall accuracy. The model obtained using this procedure was highly accurate (MSE $=.063$ ) with an average prediction accuracy of $92.5 \%$. Receiver operating characteristics (ROC) confirmed a significant effect of the averaged network output $(\mathrm{AUC}=.98, p<.001)$, as displayed in Figure 1 . 


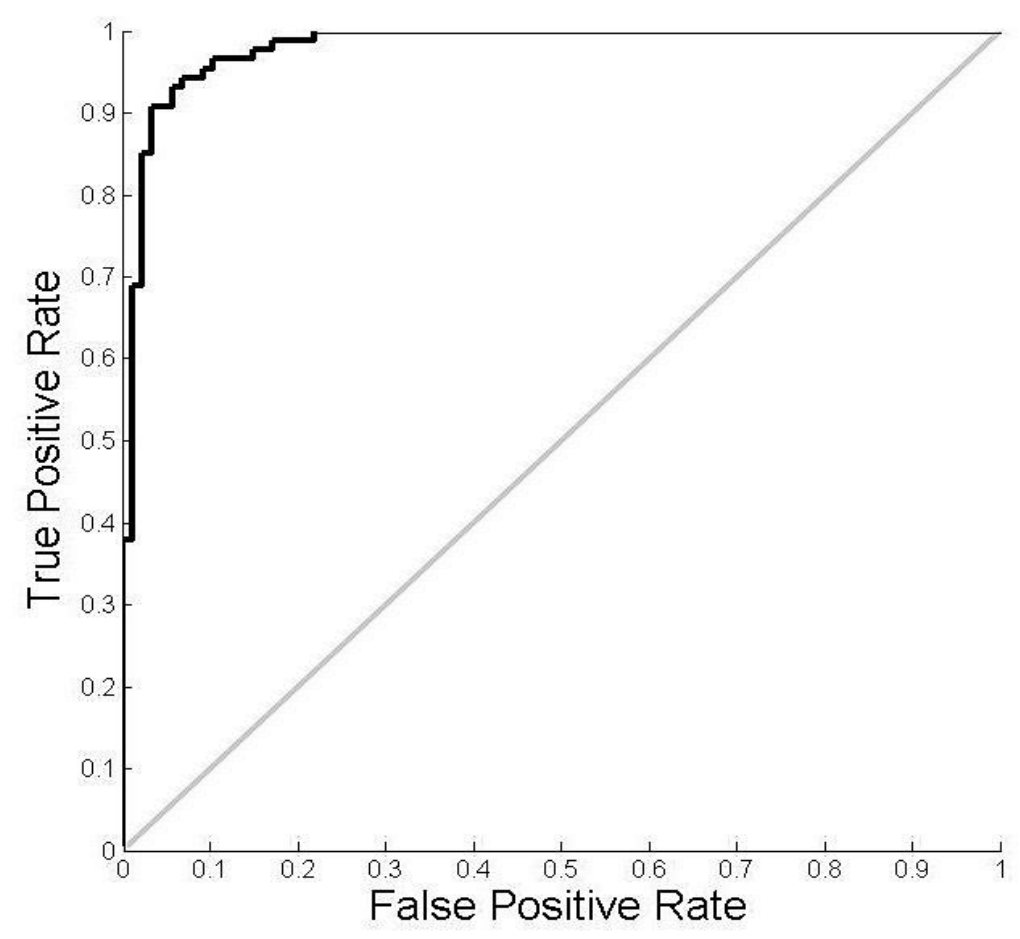

Figure 1. Receiver operating characteristics (ROC) averaged for 1000 logistic neural networks predicting geomagnetic storm/no storm; grey centre line indicates $50 \%$ chance expectations.

In order to examine simple mean comparison of significant predictor variables between days of geomagnetic storm and no-storm activity, simple independent $t$-tests were conducted for scalar IMF and SW plasma speed values.

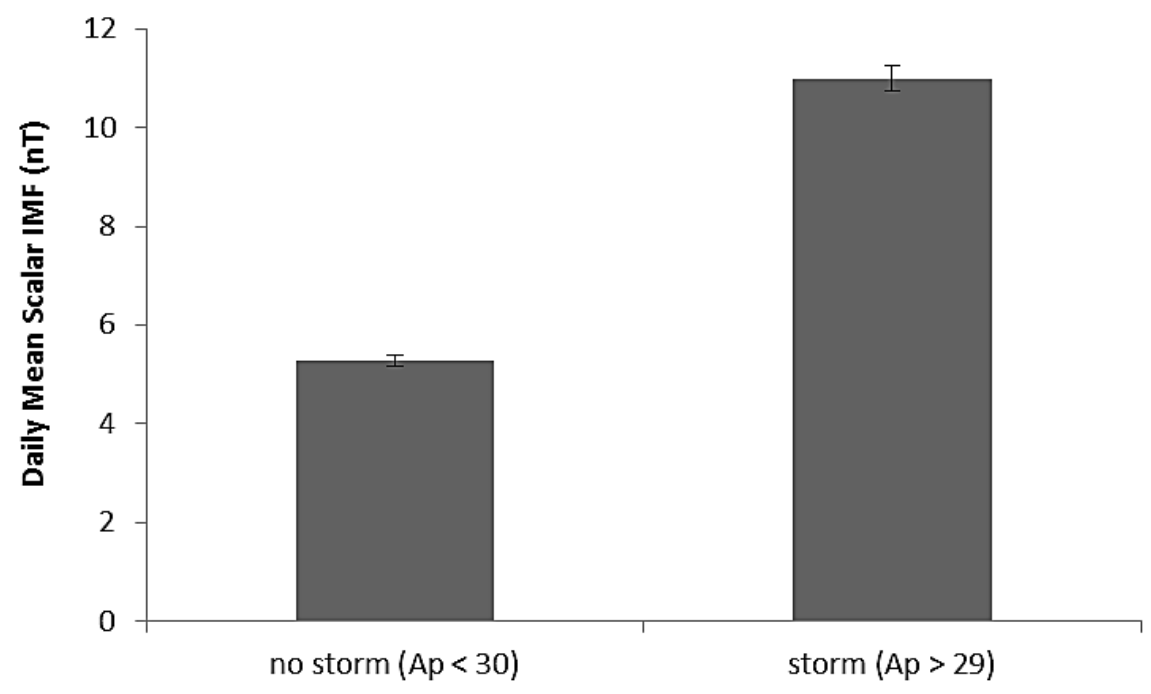

Figure 2. Average difference in the scalar component of the interplanetary magnetic field (IMF) between days of "quiet" (no storm) and storm-level daily geomagnetic activity; error bars indicate standard error of the mean. 
The scalar component of the IMF (nT) was significantly greater $\left(t_{(575)}=21.086, p<\right.$ $.001, d=1.759, r=.66)$ during days of geomagnetic storm $(\mu=10.983, \sigma=4.259)$ compared to days with relatively "quiet" activity $(\mu=5.267, \sigma=1.898)$, as displayed in Figure 2 .

Similarly, the average speed of SW plasma was also significantly greater $\left(t_{(575)}=\right.$ 15.517, $p<.001, d=1.294, r=.543)$ during geomagnetic storm days $(\mu=570.827, \sigma=$ $121.145)$ compared to geomagnetically "quiet" days $(\mu=430.4, \sigma=95.579)$, as shown in Figure 3.

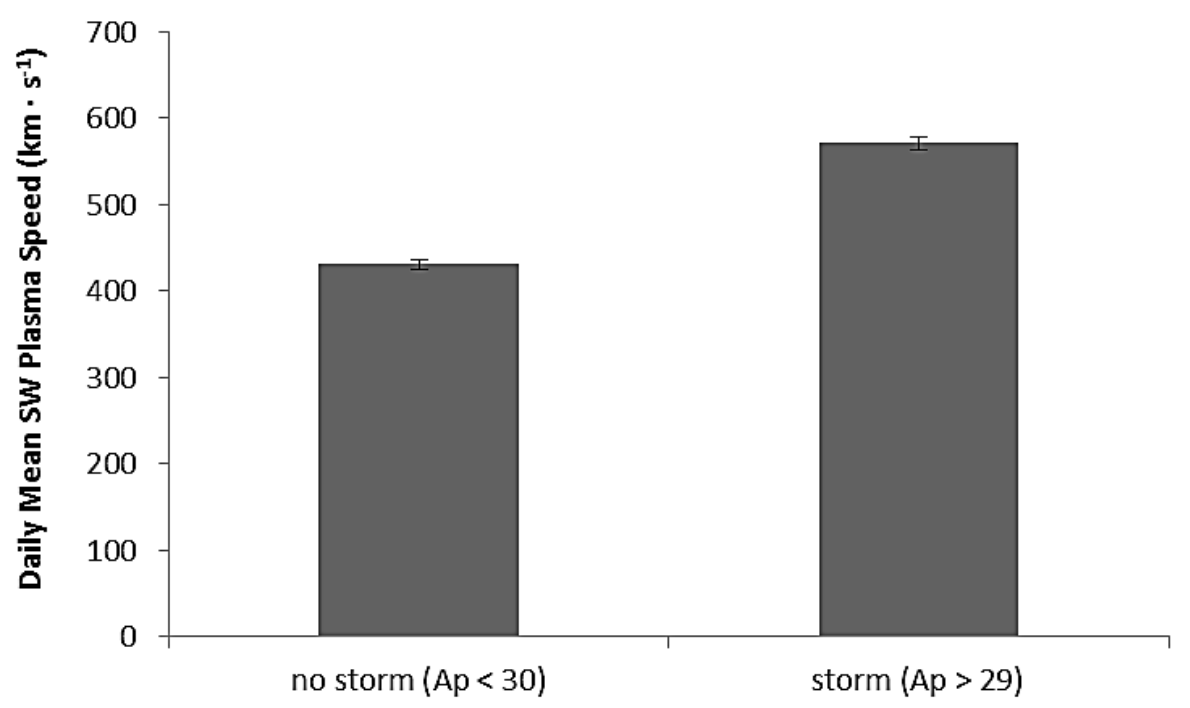

Figure 3. Average difference in solar wind (SW) plasma speed between days of "quiet" (no storm) and storm-level daily geomagnetic activity; error bars indicate standard error of the mean.

\section{CONCLUSIONS}

The results of the preceding analyses demonstrate the potential for simple binary prediction of storm-level events of the geomagnetic field using both standard and more novel statistical techniques. This study represents one of very few which has examined the potential for a logistic prediction model construction in this context [7]. Furthermore, a greater level of statistical detail for overall results is provided in the current study. Given the detailed overview of analytical techniques conducted and the freely available nature of the data used, this should easily allow independent investigation and extension of these results.

Being able to predict storm-level activity of the planetary magnetic field could have a range of implications across disciplines, from physics and astronomy to biology, particularly regarding the apparent effects of sudden increases in geomagnetic activity on the human brain [15]. That geomagnetic storms have been associated with a range of terrestrial effects [1-4] should warrant enhanced exploration and implementation of predictive models in this context.

Furthermore, the current analyses also provide additional support to the integral role of interplanetary magnetic field (IMF) and solar wind (SW) variations in the production of geomagnetic storms. As previously discussed, the role the IMF and SW [10] has been implicated in storm-level activity of the planetary magnetic field. The present study identified the IMF as well as the speed of SW as the greatest overall predictors in the context of all-or- 
none binary geomagnetic storm data, consistent with previous findings [5]. The significant differences in these variables identified between days of storm or no-storm activity suggest that the difference in interplanetary measures between geomagnetic conditions is strikingly large and readily amenable to accurate prediction. The greater overall strength of the scalar IMF component as the primary model predictor is consistent with previous geomagnetic prediction analyses [5] and supports an electromagnetic "coupling" within the geosphere.

Whereas much of the previous attention has been placed upon the destructive or deleterious consequences of an occurring geomagnetic storm, there could be biofunctional features of interactions between the IMF and the Earth's bow shock. Power spectra of the IMF vary as a function of location in space where values of $10^{-4} \mathrm{~Hz}$ to $10^{-6} \mathrm{~Hz}$ are characteristic of the field macrostructure at $>0.1$ AU [16]. Measurements at 1 AU have demonstrated similar $1 / f$ dependence within the same oscillatory range [17]. If IMF interactions with the Earth's bow shock at the boundary of the magnetopause can sufficiently increase the frequency of the local IMF, an additional implication for consciousness is proposed: A photon whose frequency is $10^{-2} \mathrm{~Hz}$ displays an intrinsic energy when multiplied by Planck's constant $\left(6.63 \cdot 10^{-34} \mathrm{~J} \cdot \mathrm{s}^{-1}\right)$, resulting in an energy of $6.63 \cdot 10^{-36} \mathrm{~J}$. The energy-tomass conversion $\left(\mathrm{E}=\mathrm{m} c^{2}\right.$ ) of said photon is equivalent to $\sim 7.38 \cdot 10^{-53} \mathrm{~kg}$, which is effectively the estimated upper limit of a non-zero rest mass photon [18]. Fairfield [19] identified permanent, transverse electromagnetic waves with 20 to 100 second periodicities in the interplanetary medium region adjacent to the Earth's bow shock. The frequency equivalent or 1/f relationship is $0.01-0.05 \mathrm{~Hz}$ or $\sim 10^{-2} \mathrm{~Hz}$. Dotta et al. [20] have argued that this finite mass is implicated in brain function and satisfies contingencies which suggest an electromagnetic basis of consciousness. At this discrete boundary condition in space, an interaction sufficient to increase local IMF frequencies from $10^{-4}$ to $10^{-2} \mathrm{~Hz}$ would effectively generate photon oscillations which could potentially influence consciousness. A binary prediction technique as outlined here can not only serve as an indicator of impending dysfunctional consequences, but a glimpse into the eventuality of subtle alterations of potential functions for photo- and magneto-receptive organisms on Earth.

\section{Acknowledgments}

The authors would like to thank David A. E. Vares for internal review. Additional thanks are also extended to Dr. Natalia Papitashvili for maintaining the NASA OMNIWeb database.

\section{References}

[1] D. H. Boteler, R. J. Pirjola, H. Nevanlinna, Advances in Space Research 22 (1998) 17-27.

[2] I. G. Usoskin, M. Korte, G. A. Kovaltsov, Geophysical Research Letters 35 (2008).

[3] E. S. Babayev, A. A. Allahverdiyeva, Advances in Space Research 40 (2007) 1941-1951.

[4] K. S. Saroka, J. M. Caswell, A. Lapointe, M. A. Persinger, Neuroscience Letters 560 (2014) 126-130.

[5] J. M. Caswell, Journal of Signal and Information Processing 5 (2014) 42-53.

[6] H. Lundstedt, H. Gleisner, P. Wintoft, Geophysical Research Letters 29 (2002) 34.1-34.4. 
[7] N. Srivastava, Annales Geophysicae 23 (2005) 2969-2974.

[8] J. Zhang, I. G. Richardson, D. F. Webb, N. Gopalswamy, E. Huttunen, J. C. Kasper, N. V., Nitta, W. Poomvises, B. J. Thompson, C. C. Wu, S. Yashiro, A. N. Zhukov, Journal of Geophysical Research: Space Physics 112 (2007).

[9] R. J. Boynton, M. A. Balikhin, S. A. Billings, O. A. Amariutei, Annales Geophysicae 31 (2013) 673-679.

[10] M. A. Persinger, International Journal of Physical Sciences 4 (2009) 44-46.

[11] B. Kirov, V. N. Obridko, K. Georgieva, E. V. Nepomnyashtaya, B. D. Shelting, Geomagnetism and Astronomy 53 (2013) 813-817.

[12] F. Faul, E. Erdfelder, A. G. Lang, A. Buchner, Behavior Research Methods 39 (2007) 175-191.

[13] F. Faul, E. Erdfelder, A. Buchner, A. G., Lang, Behavior Research Methods 41 (2009) 1149-1160.

[14] N. J. D. Nagelkerke, Biometrika 78 (1991) 691-692.

[15] B. P. Mulligan, M. A. Persinger, Neuroscience Letters 516 (2012) 54-56.

[16] J. W. Sari, N. F. Ness, Solar Physics 8 (1969) 155-165.

[17] W. H. Matthaeus, M. L. Goldstein, Physical Review Letters 57 (1986) 495-498.

[18] L. C. Tu, J. Luo, G. T. Gillies, Reports on Progress in Physics 68 (2005) 77-130.

[19] D.H., Fairfield, Journal of Geophysical Research 74 (1969), 3541-3553.

[20] B. T. Dotta, C. A. Buckner, R. M. Lafrenie, M. A. Persinger, Brain Research (2011) 77-88. 